\title{
Multiclass and multi-residue screening of mycotoxins, pharmacologically active substances, and pesticides in infant milk formulas through ultra-high-performance liquid chromatography coupled with high-resolution mass spectrometry analysis
}

\author{
Luana Izzo, ${ }^{1 *} \dagger$ Alfonso Narváez, ${ }^{1 *}$ Luigi Castaldo, ${ }^{1 *}$ Anna Gaspari, ${ }^{1}$ Yelko Rodríguez-Carrasco, ${ }^{2} \dagger$ \\ Michela Grosso, ${ }^{3,4}$ and Alberto Ritieni ${ }^{1,5}$ \\ ${ }^{1}$ Department of Pharmacy, University of Naples "Federico II," 80131 Naples, Italy \\ ${ }^{2}$ Laboratory of Food Chemistry and Toxicology, Faculty of Pharmacy, University of Valencia, 46100 Burjassot, Valencia, Spain \\ ${ }^{3}$ Department of Molecular Medicine and Medical Biotechnology, School of Medicine, University of Naples "Federico II," 80131 Naples, Italy \\ ${ }^{4}$ CEINGE-Biotecnologie Avanzate, 80131 Naples, Italy \\ ${ }^{5}$ UNESCO Chair on Health Education and Sustainable Development, "Federico II" University, 80131 Naples, Italy
}

\begin{abstract}
Infant milk formulas are designed to substitute human milk when breastfeeding is unavailable. In addition to human milk and milk-derived products, these formulas can be a vehicle of contaminants. In this work, a multiclass method based on the QuEChERS (quick, easy, cheap, effective, rugged, and safe) approach was developed for the simultaneous determination of contaminants $(\mathrm{n}=45)$, including mycotoxins and veterinary drug residues, occurring in infant milk formulas. By using an ultra-high-performance liquid chromatography quadrupole-Orbitrap coupled with high-resolution mass spectrometry analysis (UHPLCQ-Orbitrap HRMS; Thermo Fisher Scientific), further retrospective analysis of 337 contaminants, including pesticides, was achieved. The method was validated in accordance with European regulations and applied for the analysis of 54 infant milk samples. Risk assessment was also performed. Dexamethasone was detected in $16.6 \%$ of samples (range: $0.905-1.131 \mathrm{ng} / \mathrm{mL}$ ), and procaine benzyl penicillin in 1 sample at a concentration of $0.295 \mathrm{ng} / \mathrm{mL}$. Zearalenone was found in $55.5 \%$ of samples (range: $0.133-0.638 \mathrm{ng} / \mathrm{mL}$ ) and $\alpha$-zearalenol in $16.6 \%$ of samples (range: $1.534-10.408 \mathrm{ng} / \mathrm{mL}$ ). Up to 49 pesticides, 11 veterinary drug residues, and 5 mycotoxins were tentatively identified via retrospective analysis based on the mass spectral library. These findings highlight the necessity of careful evaluation of
\end{abstract}

Received August 6, 2021.

Accepted December 5, 2021.

*These authors contributed equally to this work.

†Corresponding authors: luana.izzo@unina.it and yelko.rodriguez@ uv.es contaminants in infant formulas, considering that they are intended for a vulnerable part of the population.

Key words: infant milk formula, mycotoxins, veterinary drug residues, pesticide, UHPLC-Q-Orbitrap HRMS

\section{INTRODUCTION}

Infant milk formulas (IMF) are a complementary food that occupies a special place in childhood feeding and that become an essential product when breastfeeding is unavailable. Infant milk formulas are the only processed foodstuff that totally fulfills the nutritional requirements of infants through the first months of life (de Mendonça Pereira et al., 2020; Masum et al., 2021). Worldwide recommendations for infant feeding are sole breastfeeding up to 6 mo old and nutritionally complementary feeding from 6 mo to $2 \mathrm{yr}$ of age or more (UNICEF, 2019). To safeguard infant health, it is essential to ensure that the products marketed are suitable. An adequate selection of raw materials, which includes strict limitations on contaminant residues, should be demanded in their manufacture. Infant milk formulas are principally produced based on cow milk subsequently diluted, skimmed, and enriched with minerals and vitamins (Codex Alimentarius, 2016). However, IMF can contain toxic substances due to potential contamination from the chain production of the raw material and may serve as a vehicle for the transmission of toxic substances such as mycotoxins (Becker-Algeri et al., 2016).

Mycotoxins are common secondary metabolites produced by commensal genera of filamentous fungi such as Penicillium, Aspergillus, Fusarium, Claviceps, and Alternaria. Mycotoxin contamination represents a great worldwide concern due to their serious health hazards, including mutagenicity, teratogenicity, and carcinoge- 
nicity. Even though several hundred mycotoxins have been identified to date, those responsible for common mycotoxicological risk concerns are represented by aflatoxins (AF), trichothecenes, ochratoxins, fumonisins, patulin, citrinin, and ergot alkaloids (Haque et al., 2020). Among these, AF are classified as carcinogenic to humans and included in group 1 by the International Agency for Research on Cancer; fumonisins and ochratoxins are included in group 2B and classified as possibly carcinogenic to humans (IARC, 2012). Generally, mycotoxin contamination occurs directly in the field, or during the post-harvest period, which includes processing, storage, and distribution of harvested products. In addition, when animals ingest contaminated foodstuffs, mycotoxins are metabolized, biotransformed, and secreted into animal products, such as milk or meat (Benkerroum, 2016; Narváez et al., 2020b).

Among mycotoxins, aflatoxin $\mathrm{M}_{1}\left(\mathbf{A} \mathbf{F} \mathbf{M}_{1}\right)$, the hydroxylated metabolite of $\mathrm{AFB}_{1}$, represents the mycotoxin of greatest incidence in milk and dairy products (Awaisheh et al., 2019). Moreover, when animal feed was supplemented with silage, ochratoxin, zearalenone, T2 toxin, fumonisins, and deoxynivalenol were also identified in milk and milk derivatives, although these have not been as extensively studied as $\mathrm{AFM}_{1}$ (Ogunade et al., 2018; Ushkalov et al., 2020). To protect consumers' health, the regulatory authorities set a maximum limit for $\mathrm{AFM}_{1}$ of $0.05 \mu \mathrm{g} / \mathrm{kg}$ in raw milk, heat-treated milk, and milk for the manufacture of milk-based products, restricted to $0.025 \mu \mathrm{g} / \mathrm{kg}$ for infant formulas and follow-on formulas and dietary foods for special medical purposes intended specifically for infants, under regulation no. 1881/2006 (EC, 2006b).

Another group of contaminants of relevant concern in IMF are represented by pesticides, synthetic compounds used for the management of parasites in agricultural production. These organisms can reach the food chain through consumption of contaminated fodder and pastures (Mol et al., 2008; Aguilera-Luiz et al., 2011). Several classes of pesticides, such as organo-phosphorus, pyrethroids and carbamate, and organo-chlorines, have been reported in milk and milk derivatives (Akhtar and Ahad, 2017). Milk consumption is not free from risk of exposure to pesticides, and this deserves more attention from researchers. Maximum residue levels (MRL) for pesticides have been established by the European Union in regulation no. 839/2008 (EC, 2008).

In addition, IMF may also contain residues of pharmacologically active substances. Veterinary drugs are frequently used to prevent and treat diseases in foodproducing animals. However, incorrect use or noncompliance with the withdrawal period after treatment may allow release of residues from active substances that, after accumulation in the body, lead to injurious effects in humans (Becker-Algeri et al., 2016). The normal use of veterinary drugs to control animal diseases or improve production efficiency is acceptable when meeting the MRL tolerated in food products set in regulation EC 37/2010 by the Codex Alimentarius Commission (EC, 2009). However, the withdrawal period that must elapse between the administration of the veterinary drug and the recovery of raw material for food production intended for human consumption is set by directive 2004/28/CE (EC, 2004).

To guarantee effective consumer safety, reliable methods have been validated for the analysis of contaminants in food matrices (Barreiro et al., 2015; Thompson and Darwish, 2019). Until now, few scientific works have reported methods for the simultaneous analysis of pharmacologically active substances, pesticides, and mycotoxins in milk (Zhan et al., 2012; Danezis et al., 2016; Zhou et al., 2018; Izzo et al., 2020). The development of an optimal method should allow good recovery of the analytes and good reproducibility of the data, and, at the same time, it should be fast, easy to reproduce, and guarantee lower toxic organic solvent consumption.

Among the various available method approaches used in the pretreatment of samples, the QuEChERS (quick, easy, cheap, effective, rugged, and safe) approach represents the one that comes closest to achieving the characteristics mentioned above. It is largely employed to extract different groups of compounds and represents the most frequently used pretreatment technique in foods analysis (González-Curbelo et al., 2015; Rossi et al., 2018; Samsidar et al., 2018; Narváez et al., 2020a). The most frequent analytical methods are based on liquid chromatography coupled to MS. The use of ultra-high-performance liquid chromatography (UHPLC) provides higher sensitivity, a reduction in mobile phase consumption, and an increase in resolving power and peak shape. High-resolution mass spectrometry (HRMS) provides sensitive and specific measurements for the quantification of targeted compounds, with the additional features of retrospective data analysis and identification of untargeted compounds based on exact mass measurements (Kaufmann, 2014; López-Ruiz et al., 2019; Castaldo et al., 2020).

Bearing in mind the lack of studies reporting multiclass analysis, the current scientific study aimed (1) to develop a multi-residue method for the identification of target mycotoxins and pharmacologically active substances $(\mathrm{n}=45)$ in IMF through a QuEChERS-based extraction coupled to UHPLC quadrupole (Q)-Orbitrap HRMS (Thermo Fisher Scientific); (2) to apply the developed method for evaluating the occurrence in 
54 samples collected from the Italian market; and (3) to detect possible untargeted compounds $(\mathrm{n}=337)$, including pesticides, through retrospective analysis.

\section{MATERIALS AND METHODS}

\section{Chemicals and Materials}

Methanol, acetonitrile, and water for chromatography (LC-MS grade) were purchased from Merck. Ammonium formate $\left(\mathrm{NH}_{4} \mathrm{HCO}_{2}\right.$, analytical grade) and formic acid ( $\mathrm{HCOOH}, \mathrm{MS}$ grade) were acquired from Fluka. Sodium chloride $(\mathrm{NaCl})$, anhydrous sulfate sodium $\left(\mathrm{Na}_{2} \mathrm{SO}_{4}\right)$, anhydrous sodium acetate $\left(\mathrm{C}_{2} \mathrm{H}_{3} \mathrm{NaO}_{2}\right)$, primary secondary amine sorbent (PSA), and discovery octadecyl silica $\left(\mathrm{C}_{18}\right.$, analytical grade) were supplied by Sigma-Aldrich.

Conical polypropylene centrifuge and microcentrifuge tubes and polypropylene syringes without needles were acquired from Microtech Srl. Syringe filters with a nylon membrane (Phenex-NY 15-mm syringe filters, $0.2-\mu \mathrm{m}$ diameter) and glass amber vials with septum screw caps were supplied by Phenomenex.

Standards of mycotoxins and pharmacologically active substances (purity $>98 \%$ ) were obtained from Sigma-Aldrich. European Commission regulation no. $37 / 2010$ (EC, 2009) was used as a starting point to select pharmacologically active substances with lower MRL or those that are prohibited in milk.

The acquired pharmacologically active substances $(\mathrm{n}=23)$ standards included abamectin, amoxicillin, ampicillin, ceftiofur, chloramphenicol, clenbuterol hydrochloride, cyhalothrin, cypermethrin, colchicine, danofloxacin, dapsone, dexamethasone, deltamethrin, doramectin, eprinomectin, imidocarb, ivermectin, metronidazole, meloxicam, monensin sodium, procaine benzylpenicillin, sulfadimidine, and trichlorfon.

The acquired mycotoxin $(\mathrm{n}=22)$ standards included aflatoxins $\left(\mathrm{AFM}_{1}, \mathrm{AFB}_{1}, \mathrm{AFB}_{2}, \mathrm{AFG}_{1}\right.$, and $\left.\mathrm{AFG}_{2}\right)$, alternariol, alternariolmonomethyl ether, beauvericin, deoxynivalenol, enniatins (ENNA, ENNA1, ENNB, and ENNB1), fusarenon-X, neosolaniol (NEO), HT2 toxin, T2 toxin, zearalanol (ZEN), $\alpha$ - and $\beta$-zearalenol $(\alpha-$ and $\beta$-ZEL), $\alpha$-zearalanol, and $\beta$-zearalanol.

The individual standard solution of each analyte was prepared in the optimal solvent recommended by the supplier at a concentration of $1 \mathrm{mg} / \mathrm{mL}$. After that, 2 stock solutions were prepared: stock solution 1, which included all mycotoxins, and stock solution 2, which included the remaining pharmacologically active compounds. These stock solutions were optimally diluted in methanol to obtain appropriate working standard solutions to use in spiking tests. The working standard solutions were prepared at $0.5,5$, and $25 \mathrm{ng} / \mathrm{mL}$. Stock solutions were kept at $-20^{\circ} \mathrm{C}$ in screw-capped glass vials.

\section{Sampling}

Three lots of 18 different brands of IMF were purchased from supermarkets in the Campania region, in southern Italy. The analysis was performed on a total of 54 samples. Samples included infant milk powder $(\mathrm{n}=18)$ and liquid infant milk $(\mathrm{n}=36)$ commonly consumed in childhood. Samples were kept in their original packages and stored at $4^{\circ} \mathrm{C}$ until analysis, which wasperformed within $48 \mathrm{~h}$ after their arrival in the laboratory.

\section{Sample Preparation}

Extraction was performed in accordance with the procedure described by Izzo et al. (2020), with slight modifications. Aliquots of $1.4 \mathrm{~g}$ of infant milk powder were weighed into $50-\mathrm{mL}$ propylene tubes (Conical Polypropylene Centrifuge Tube, Thermo Fisher Scientific) and dissolved with $10 \mathrm{~mL}$ of pure water as recommended by the manufacturer. In short, to $10 \mathrm{~mL}$ of the liquid sample, $2.5 \mathrm{~mL}$ of distilled water and 5 $\mathrm{mL}$ of acetonitrile containing $3.35 \%$ formic acid ( $\mathrm{vol} /$ vol) were added. The $50-\mathrm{mL}$ Falcon tube was vortexed (ZX3, VEPL Scientific) for $1 \mathrm{~min}$ and sonicated (LBS 1, Zetalab SRL) for $15 \mathrm{~min}$. A mixture of salts, which included $4.0 \mathrm{~g}$ of anhydrous sulfate sodium, $1.2 \mathrm{~g}$ of sodium chloride, and $0.5 \mathrm{~g}$ of anhydrous sodium acetate, was added. This step was followed by manual shaking for $1 \mathrm{~min}$ and centrifugation (X3R Heraeus Multifuge, Thermo Fisher Scientific) for $3 \mathrm{~min}$ at $1,792 \times g$ and $4^{\circ} \mathrm{C}$. After centrifugation, all the supernatant was recovered and transferred into a $15-\mathrm{mL}$ Falcon tube in which $300 \mathrm{mg}$ of $\mathrm{C} 18$ sorbent, $140 \mathrm{mg}$ of PSA, and $1.5 \mathrm{~g}$ of anhydrous sulfate sodium were weighed. The $15-\mathrm{mL}$ falcon tube was vortexed for $1 \mathrm{~min}$ and centrifuged at $4^{\circ} \mathrm{C}$ and $252 \times g$ for $1 \mathrm{~min}$. The upper layer was collected into a clear $15-\mathrm{mL}$ Falcon tube and dried under gentle nitrogen flow at $45^{\circ} \mathrm{C}$. The residue was reconstituted with $500 \mu \mathrm{L}$ of methanol:water (70:30, vol/vol), filtered through a $0.22-\mu \mathrm{m}$ filter, and transferred into an amber vial for the UHPLC-Q-Orbitrap HRMS analysis.

\section{UHPLC-Q-Orbitrap HRMS Analysis}

Analysis was performed as previously described by Izzo et al. (2020), with some modifications. For the qualitative and quantitative analyses of compounds, a UHPLC (Dionex UltiMate 3000, Thermo Fisher Scientific) equipped with a degassing system, a quaternary UHPLC pump working at $125 \mathrm{MPa}$, and a refriger- 
ated autosampler device were used. Chromatographic separation was carried out with a thermostated $(\mathrm{T}=$ $\left.25^{\circ} \mathrm{C}\right)$ Kinetex Biphenyl 2.6- $\mu \mathrm{m}$ column $(100 \times 2.1 \mathrm{~mm}$, Phenomenex $)$ coupled to a guard column $(5 \times 2 \mathrm{~mm}$, $1.8-\mu \mathrm{m}$ particle size) and an inline filter. The mobile phase consisted of water containing $5 \mathrm{mM}$ ammonium acetate (A) and methanol containing $5 \mathrm{~m} M$ ammonium acetate (B). The injection volume was $5 \mu \mathrm{L}$, and the flow rate was $0.5 \mathrm{~mL} / \mathrm{min}$. Separation was achieved under the following gradient conditions: 0 to $0.5 \mathrm{~min}, 10 \%$ B; 0.5 to $2.5 \mathrm{~min}, 10$ to $80 \% \mathrm{~B} ; 2.5$ to $5.5 \mathrm{~min}, 100 \% \mathrm{~B}$; 5.5 to $7.5 \mathrm{~min}, 100$ to $10 \% \mathrm{~B}$; and then the column was re-equilibrated at $10 \% \mathrm{~B}$ for $1.5 \mathrm{~min}$ before the next injection.

The Q-Orbitrap HRMS system was operated in both positive and negative ionization modes. Full scan and data-independent all-ion fragmentation spectra were collected.

The following scan parameters were used in full MS mode: mass resolution power of 35,000 full width at half maximum, scan range 90 to $1,000 \mathrm{~m} / z$, automatic gain control target $1 \times 10^{6}$, maximum inject time set to $200 \mathrm{~ms}$, and scan rate $3 \mathrm{scan} / \mathrm{s}$. The ion source parameters were as follows: sheath gas $\left(\mathrm{N}_{2}>95 \%\right) 35$, auxiliary gas $\left(\mathrm{N}_{2}>95 \%\right) 10$, spray voltage $2.8 \mathrm{kV}$; capillary temperature $310^{\circ} \mathrm{C}$; S-lens $\mathrm{RF}$ level 50; and auxiliary gas heater temperature $305^{\circ} \mathrm{C}$.

The following scan parameters were used in all-ion fragmentation mode: mass resolution power of 17,500 full width at half maximum, scan range $80-1,000 \mathrm{~m} / z$, automatic gain control target $1 \times 10^{5}$, maximum inject time set to $200 \mathrm{~ms}$, and scan rate $3 \mathrm{scan} / \mathrm{s}$; scan time $=0.10 \mathrm{~s}$; isolation window $5.0 \mathrm{~m} / z$; and retention time $30 \mathrm{~s}$. The collision energy was varied in the range of 10 to $45 \mathrm{eV}$ to obtain representative product ion spectra. A mass tolerance below $2 \mathrm{ppm}$ was set for the identification of the molecular ion and fragments. Data processing was performed by using Xcalibur software, version 3.1.66.10 (Thermo Fisher Scientific; RodríguezCarrasco et al., 2018).

\section{Retrospective Screening}

Retrospective analysis was carried out as previously described by Izzo et al. (2020), with slight modifications. Qualitative analysis of compounds for which a reference standard was not available was carried out by retrospective analysis of the data. The retrospective analysis of data was performed on spectral data collected using 3 spectral libraries (Mycotoxin Spectral Library version 1.1 for LibraryView Software, AB Sciex; Antibiotics Spectral Library version 1.0 for LibraryView Software, AB Sciex; and Pesticide Spectral Library version 1.1 for LibraryView Software, AB
Sciex). Identification was performed by searching in the extract for the exact mass to the fifth decimal place. The sample was considered suspect positive for the occurrence of a specific analyte if a peak was identified by setting a mass tolerance of $1 \mathrm{ppm}$ for the molecular ion.

\section{Validation of the Method}

Validation of the method was performed as previously described by Narváez et al. (2020b) with modifications. An analytical method for the determination of both mycotoxins and veterinary drug residues in IMF was validated in-house in accordance with European regulations (EC, 2002; EC, 2006c; European Commission Health and Food Safety Directorate General, 2016). Data quality was verified using a comprehensive range of quality guarantee and quality control procedures. To achieve a rigorous control, the in-house validation method included a reagent blank, a procedural blank, a triplicate sample, a matrix-matched calibration in each batch of samples, and 3 spiking levels. The following analytical performance parameters were evaluated: linearity, matrix effect, specificity, trueness, precision (repeatability and intermediate precision), and sensitivity expressed in terms of limit of detection, limit of quantification (LOQ), decision limits $(\mathbf{C C} \boldsymbol{\alpha})$, and detection capability $(\mathbf{C C} \boldsymbol{\beta})$.

Linearity was evaluated using solvent and matrixmatched calibration curves, injecting in triplicate concentration levels ranging from 0.0049 to $25 \mathrm{ng} / \mathrm{mL}$.

Signal suppression or enhancement effect was evaluated through a comparison between the slope of matrix-matched standard curve (A) and the slope of pure standard curve (B), calculated as the ratio (A/B $\times 100)$. Signal suppression occurred if the value was lower than $100 \%$ and a signal enhancement if the value was higher than $100 \%$. A value of $100 \%$ indicated no matrix effect.

Specificity was evaluated by injecting a blank milk extract 10 times to confirm the absence of target analytes and to evaluate possible sample interference.

Trueness was assayed by the addition of known quantities of the studied analytes at $0.5,1$, and $5 \mathrm{ng} /$ $\mathrm{mL}$ to a blank milk sample, and was expressed as the percentage of recovery.

Precision of the validated method was determined by repeated measurements of the 3 fortification levels, on the same day (repeatability, $\mathrm{n}=9$ ) and on 3 nonconsecutive days (reproducibility, $\mathrm{n}=27$ ), and was expressed as relative standard deviation [\% RSD; intra-day (RSDr, \%) and inter-day precision (RSDR, $\%)]$.

Sensitivity was evaluated by limit of detection and LOQ. The limit of detection was established as the 
minimum concentration that allows molecular ion identification with a mass error below $2 \mathrm{ppm}$. The LOQ referred to the lowest concentration of the analyte that generates a chromatographic peak with a precision and accuracy $<20 \%$. For confirmation criteria, the retention times of analytes in standards and samples were compared (tolerance of $\pm 2.5 \%$ ).

In the case of substances for which MRL was fixed, the CC $\alpha$ values were determined by analyzing 20 blank samples fortified at the corresponding permitted limit (CMRL). Detection capability was the value of the CMRL plus 1.64 times the corresponding standard deviation (SD) of the within-laboratory reproducibility $(\alpha=5 \%)$ :

$$
\mathrm{CC} \alpha=\mathrm{CMRL}+1.64 \times \mathrm{SD}_{20 \text { representative MRL spike }}
$$

In the case of substances for which MRL was not established, $\mathrm{CC} \alpha$ was calculated as 3 times the signal-tonoise ratio $(\mathrm{S} / \mathrm{N})$ of the baseline of 20 representative blank samples:

$$
\mathrm{CC} \alpha=3 \mathrm{~S} / \mathrm{N}_{20} \text { representative blank samples. }
$$

The CC $\beta$ was estimated by analyzing 20 blank samples fortified at the previously estimated $\mathrm{CC} \alpha$ decision limit $(\beta=5 \%)$ :

$$
\mathrm{CC} \beta=\mathrm{CC} \alpha+1.64 \times \mathrm{SD}_{20} \text { representative spike at } \mathrm{CC} \alpha \text { level } .
$$

\section{Statistical Analysis}

Sample analysis was performed in triplicate and results expressed as mean \pm RSD. Statistical analysis of data was performed using the software Info-Stat version 2008 (https://www.infostat.com.ar/index.php?mod = page\&id $=15)$. The level of $P \leq 0.05$ was considered statistically significant.

\section{RESULTS AND DISCUSSIONS}

\section{Optimization UHPLC-Q-Orbitrap HRMS Conditions}

During the method development stage, each of 45 standards were infused at a concentration of $1 \mu \mathrm{g} / \mathrm{mL}$ into the Q-Orbitrap system using syringe injection at a flow rate of $10 \mu \mathrm{L} / \mathrm{min}$. The obtained MS parameters ensured assignment of the correct identities to the respective compounds in a mixture of standards. The instrument was operated in the positive and negative electrospray modes. For the studied analytes, the best fragmentation patterns were obtained in positive electrospray mode, producing the quasi-molecular ion $[\mathrm{M}+\mathrm{H}]^{+}$. Due to the presence of ammonium formate in the mobile phases, the compounds ivermectin, cyhalothrin, cypermethrin, deltamethrin, abamectin, doramectin, NEO, T2 and HT2, enniatins, and beauvericin exhibited ammonium adduct species as the most predominant ions in the mass spectrum (Jia et al., 2014; Gómez-Pérez et al., 2015; Castaldo et al., 2021).

The collision energy of each studied compound was carefully set to obtain the best fragmentation patterns. The MS parameters for all compounds are shown in Table 1. For accurate mass measurement, identification and confirmation were performed at a mass tolerance of $2 \mathrm{ppm}$ for the precursor molecular ion and for relative fragments.

In this experiment, the column chosen for chromatography separation reached superior resolution, speed, and sensitivity compared with another column tested in a previous study (Izzo et al., 2020). A first comparison was made by comparing a Luna Omega column $(50 \times 2.1 \mathrm{~mm}, 1.6-\mu \mathrm{m}$; Phenomenex $)$ used in the chromatographic separation of our previously published study with that of the present study's Kinetex Biphenyl 2.6- $\mu \mathrm{m}(100 \times 2.1 \mathrm{~mm}$, Phenomenex $)$. The characteristics of the Kinetex Biphenyl column allowed a suitable separation of hydrophobic and hydrophilic compounds simultaneously. The ability to reach $100 \%$ aqueous mobile phase in the initial run time was useful for competent retention of hydrophilic analytes. Optimal results in terms of retention time, good peak shape, and chromatographic separation of the analytes were obtained with this chromatographic column. Moreover, ammonium formate was added to both mobile phases to help reduce the formation of unwanted adducts and stabilize the $\mathrm{pH}$ (Chung and Lam, 2015; Wittenberg et al., 2017).

Having made the choice of the column, different gradients were tested to obtain the best chromatographic separation of all analytes. Chromatographic separation was evaluated under the following gradient conditions:

- Gradient 1: 0 to $1.5 \mathrm{~min}, 0 \% \mathrm{~B} ; 0.5$ to $3.5 \mathrm{~min}, 10$ to $50 \% \mathrm{~B} ; 3.5$ to $6.5 \mathrm{~min}, 100 \% \mathrm{~B}$; 6.5 to $9.5 \mathrm{~min}$, 100 to $0 \% \mathrm{~B}$; column re-equilibrated at $10 \% \mathrm{~B}$ for 2.5 min before the next injection; total run time $12 \mathrm{~min}$;

- Gradient 2: 0 to $1.5 \mathrm{~min}, 10 \% \mathrm{~B} ; 0.5$ to $2.5 \mathrm{~min}, 10$ to $80 \% \mathrm{~B} ; 2.5$ to $5.5 \mathrm{~min}, 100 \% \mathrm{~B} ; 5.5$ to $9.5 \mathrm{~min}$, 100 to $0 \% \mathrm{~B}$; column re-equilibrated at $10 \% \mathrm{~B}$ for $1.5 \mathrm{~min}$ before the next injection; total run time $12 \mathrm{~min}$;

- Gradient 3: 0 to $0.5 \mathrm{~min}, 10 \% \mathrm{~B}$; 0.5 to $2.5 \mathrm{~min}, 10$ to $80 \% \mathrm{~B} ; 2.5$ to $5.5 \mathrm{~min}, 100 \% \mathrm{~B} ; 5.57 .5 \mathrm{~min}, 100$ to $10 \% \mathrm{~B}$; column re-equilibrated at $10 \% \mathrm{~B}$ for $1.5 \mathrm{~min}$ before the next injection; total run time 9 min. 
Table 1. Chromatographic retention time and optimized MS/MS parameters for 45 target analytes in commercial infant milk formula samples

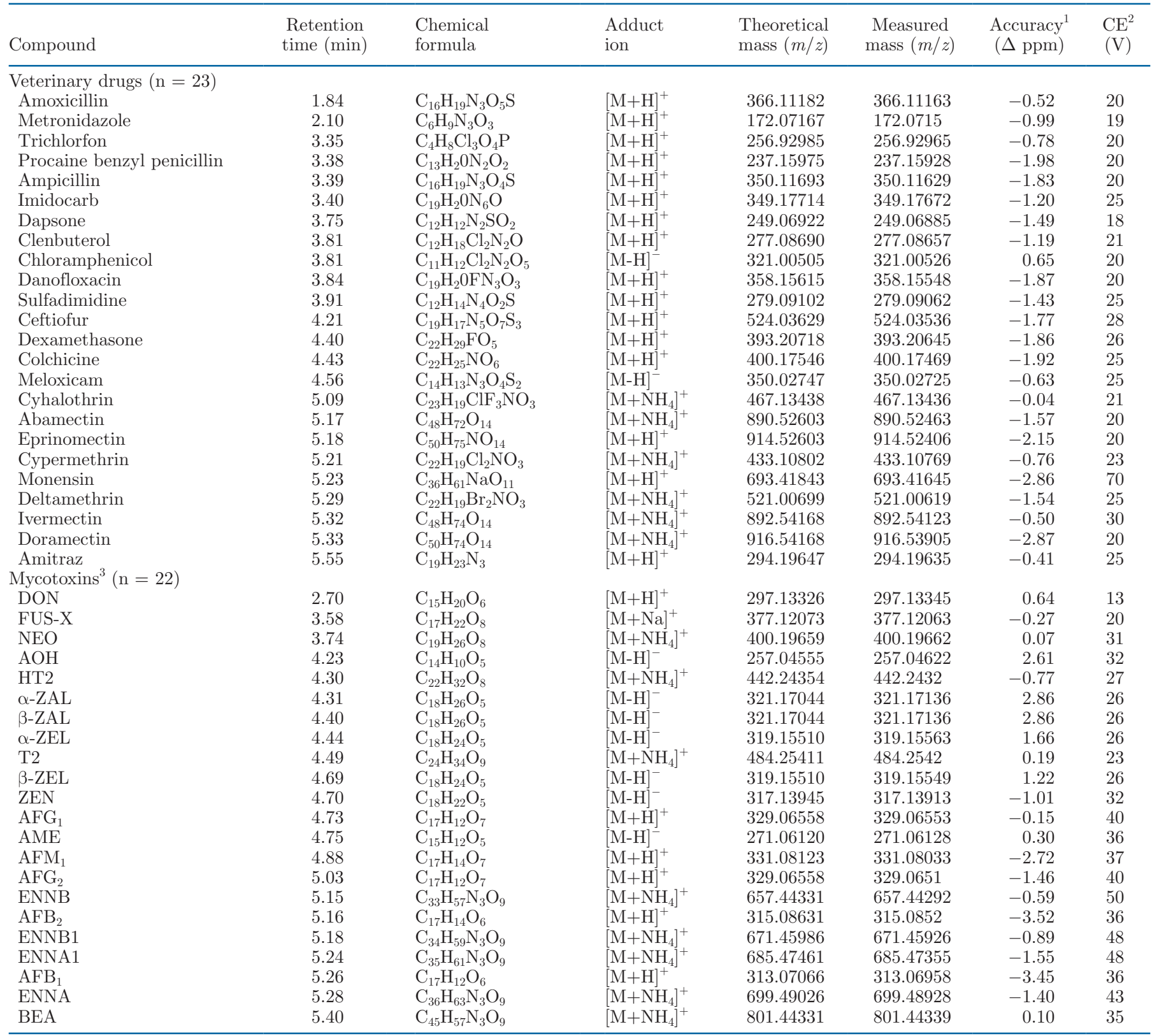

${ }^{1}$ Accuracy $=[($ measured mass $m / z$ - theoretical mass $m / z) /$ theoretical mass $m / z] \times 10^{6}=\mathrm{ppm}$.

${ }^{2} \mathrm{CE}=$ collision energy.

${ }^{3}$ Mycotoxin standards were as follows: aflatoxins $\left(\mathrm{AFM}_{1}, \mathrm{AFB}_{1}, \mathrm{AFB}_{2}, \mathrm{AFG}_{1}\right.$, and $\left.\mathrm{AFG}_{2}\right)$, deoxynivalenol (DON), HT2 toxin, T2 toxin, neosolaniol (NEO), fusarenon-X (FUS-X), zearalanol (ZEN), $\alpha$-zearalenol ( $\alpha$-ZEL), $\beta$-zearalenol ( $\beta$-ZEL), $\alpha$-zearalanol $(\alpha$-ZAL), $\beta$-zearalanol $(\beta-Z A L)$, beauvericin (BEA), enniatins (ENNA, ENNA1, ENNB, and ENNB1), alternariol (AOH), and alternariolmonomethyl ether (AME).

Using gradient 1 , some compounds were not retained by the stationary phase and were eluted within the dead time of the chromatographic separation. The total run time was 12 min, which led to greater organic solvent consumption. With gradient 2 , compounds were eluted between 3.5 and $7 \mathrm{~min}$, although peak response for some analytes was not regular. For gradient 3, good separation and peak shape were obtained for all studied analytes (Table 1 ). The retention times were reproducible under \pm 0.2 min for most of the target analytes.

\section{Optimization of Sample Preparation}

In this work, critical extraction parameters such as percentage of acidification and clean-up stage were evaluated. In total, 3 different sample preparation pro- 
cedures were tested for the determination of different compounds in IMF. Although the extraction process has as its starting point our previously optimized scientific on milk, because IMF represents a different matrix, a new validation is required to ensure that the developed methodology is suitable and meets the requirements of the European regulation in force. Using the protocol reported in the Sample Preparation of the Materials and Methods section as a starting point, slight changes in mixture acidification were performed. The percentage of acetonitrile acidification was 3.35\% formic acid (vol/ vol) in the first procedure, $4 \%$ in the second, and $1 \%$ in the third protocol. Optimal conditions in terms of peak shape for all the target analytes were reached by using acetonitrile containing $3.35 \%$ formic acid (vol/vol). Data regarding recovery and matrix effect were comparable between the first 2 , whereas the third protocol showed unsatisfactory results. In addition, 2 different clean-up stages were tested: the first foresaw $300 \mathrm{mg}$ of $\mathrm{C} 18$ sorbent, $140 \mathrm{mg}$ of PSA, and $1.5 \mathrm{~g}$ of anhydrous sulfate sodium, and the second $100 \mathrm{mg}$ of C18 sorbent, $500 \mathrm{mg}$ of PSA, and $1.5 \mathrm{~g}$ of anhydrous sulfate sodium. The conditions for the second clean-up were not suitable to reach appropriate recovery values $(<50 \%)$. Moreover, interference from the matrix was observed for all studied compounds (percentage of signal suppression or enhancement effect outside the range $80-120 \%$ ). Special attention is given to the various adsorbent phases used that strictly depend on the matrix. Primary secondary amine (PSA) is a common sorbent used to remove fatty acids, organic acids, lipids, and sugars from the preliminary extract. Sometimes the clean-up step with PSA sorbent may retain analytes and lead to recoveries below 50\% (Lehotay et al., 2005). Octadecyl silica (C18) provides optimal results in the purification of samples with significant fat content; at the same time, recoveries of the more lipophilic pesticides may suffer (Rejczak and Tuzimski, 2015). The clean-up of the first protocol represents a fair compromise between matrix effect and recovery values.

\section{Analytical Features of the Proposed Method}

Results of the in-house validation method for the determination of 45 analytes in milk samples are reported in Table 2. Correlation coefficients (r) $>0.990$ were obtained within the range from 0.0049 to $25 \mathrm{ng} /$ $\mathrm{mL}$. The matrix effects were in the range from $78 \%$ to $116 \%$, resulting in accordance with the limits reported by EC regulations (EC, 2002; EC, 2006c; European Commission Health and Food Safety Directorate General, 2016). The pure standard calibration curves were used for quantification purposes. As established by Commission decision 2002/657/EC, the values of recoveries at each tested spiked level ranged between $83 \%$ and $118 \%$, and the method was repeatable (RSD $<19 \%$ ) and reproducible (RSD <16\%). As regards specificity, the instrumentation used showed no signal interferences in the blank matrix for any target studied analytes. As regards sensitivity, the LOQ obtained ranged from 0.049 to $0.390 \mathrm{ng} / \mathrm{mL}$. Both CC $\beta$ and CC $\alpha$ were also determined, and results are shown in Table 3. The developed method met the requirements set in European regulations and proved to be suitable for the determination of studied analytes in IMF.

\section{Application to Samples: Target Mycotoxins and Pharmacologically Active Substances}

Substances in Milk Samples. To demonstrate the suitability of the validated method, it was applied to the analysis of 3 lots of 18 IMF brands acquired from different supermarkets in the Campania region of southern Italy. The results reported in this study referred to the average obtained from analysis of 3 different lots for each brand available in Campania supermarkets $(\mathrm{n}=$ 54 samples). Although most studies on milk have focused on evaluating occurrence of $\mathrm{AFM}_{1}$, in the last few years researchers have extended the analysis to other mycotoxins (Benkerroum, 2016; Ushkalov et al., 2020).

In this work, IMF tested positive for 2 veterinary drug residues, dexamethasone $(16.6 \%, \mathrm{n}=9)$ and procaine benzyl penicillin $(5.5 \%, \mathrm{n}=3)$, and tested positive for up to 6 mycotoxins produced by Fusarium species, including emerging mycotoxins (ENN; Table 4).

Similarly, the presence of up to 7 pharmacologically active substances, including benzylpenicillin procaine and dexamethasone, in Italian milk samples $(\mathrm{n}=56)$ has been previously reported (Izzo et al., 2020). Particularly, procaine benzyl penicillin was found in $8.9 \%$ of samples at a concentration range between 1 and $4.530 \mathrm{ng} / \mathrm{mL}$ and dexamethasone in $1.7 \%$ of samples at a concentration of $0.140 \mathrm{ng} / \mathrm{mL}$. In that case, the concentration of procaine benzyl penicillin found in one sample was slightly higher than the MRL $(4 \mu \mathrm{g} / \mathrm{kg})$. In our study, the concentration of $0.295 \mathrm{ng} / \mathrm{mL}$ did not exceed the EU limit.

Antibiotics are drugs widely used for therapeutic purposes and promotion of growth. Around $80 \%$ of food animals receive antibiotics for part or most of their lifetime. Veterinary drug residues remain one of the major concerning issues that affect the safety of the dairy industry (Bacanlı and Başaran, 2019). Dexamethasone is a potent corticosteroid indicated for the treatment of ketosis in postpartum dairy cows and as an anti-inflammatory drug in bovines but also used as an illegal growth promoter in livestock production (Shamay et al., 2000). 
Among mycotoxins, the occurrence of ZEN and its metabolite, $\alpha$-ZEL, have frequently been reported in feed components in dairy cattle diets. Mycotoxins are natural contaminants known to adulterate a wide heterogeneity of feed ingredients and final products (Brodehl et al., 2014). In this study, ZEN was the most common detected mycotoxin $(55.5 \%, \mathrm{n}=30)$, at concentrations ranging between 0.133 and $0.638 \mathrm{ng} / \mathrm{mL}$.
Although ZEN is not one of the main mycotoxins occurring in dairy products, several scientific studies have reported ZEN contamination (Guan et al., 2011; Li et al., 2014; Neme and Mohammed, 2017). Pleadin et al. (2017) analyzed concentrated dairy cattle feeds $(\mathrm{n}=$ 56), cow milk samples $(\mathrm{n}=105)$, and maize silage $(\mathrm{n}=$ 21) from Croatia and detected the occurrence of ZEN in $9.5 \%$ of maize silage samples and in $94.3 \%$ of milk

Table 2. Method performance: linearity, matrix effect (signal suppression or enhancement, SSE \%), recovery, and limit of quantification (LOQ, $\mathrm{ng} / \mathrm{mL}$ ) for 45 targeted analytes in commercial infant milk formula samples ${ }^{1}$

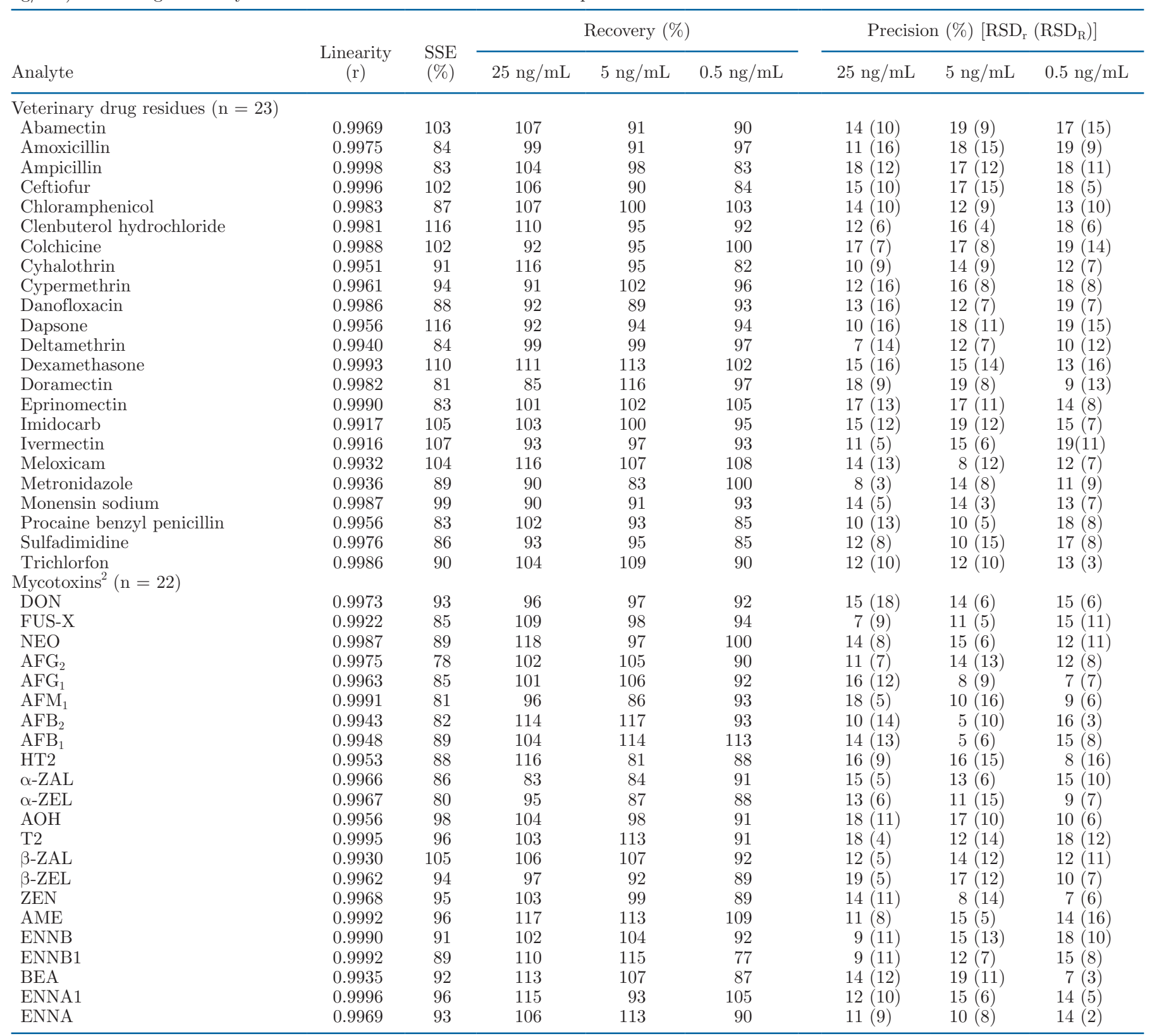

${ }^{1} \mathrm{RSD}_{\mathrm{r}}=$ intra-day relative standard deviation; $\mathrm{RSD}_{\mathrm{R}}=$ inter-day standard deviation.

${ }^{2}$ Mycotoxin standards were as follows: aflatoxins ( $\mathrm{AFM}_{1}, \mathrm{AFB}_{1}, \mathrm{AFB}_{2}, \mathrm{AFG}_{1}$, and $\left.\mathrm{AFG}_{2}\right)$, deoxynivalenol (DON), HT2 toxin, T2 toxin, neosolaniol (NEO), fusarenon-X (FUS-X), zearalanol (ZEN), $\alpha$-zearalenol $(\alpha$-ZEL), $\beta$-zearalenol $(\beta$-ZEL), $\alpha$-zearalanol $(\alpha$-ZAL), $\beta$-zearalanol $(\beta-Z A L)$, beauvericin (BEA), enniatins (ENNA, ENNA1, ENNB, and ENNB1), alternariol (AOH), and alternariolmonomethyl ether (AME). 
Table 3. Limit of quantification (LOQ), decision limit (CC $\alpha$ ), and detection capability (CC $\beta$ ) obtained for mycotoxins and veterinary drug residues in infant milk formulas, using ultra-high-performance liquid chromatography quadrupole-Orbitrap high-resolution MS (Thermo Fisher Scientific)

\begin{tabular}{|c|c|c|c|}
\hline Analyte & LOQ (ng/mL) & $\mathrm{CC} \alpha(\mathrm{ng} / \mathrm{mL})$ & $\mathrm{CC} \beta(\mathrm{ng} / \mathrm{mL})$ \\
\hline \multicolumn{4}{|c|}{ Veterinary drug residues $(\mathrm{n}=23)$} \\
\hline Abamectin & 0.390 & 0.224 & 0.257 \\
\hline Amoxicillin & 0.390 & 4.114 & 4.238 \\
\hline Ampicillin & 0.390 & 4.082 & 4.137 \\
\hline Ceftiofur & 0.390 & 101.047 & 101.380 \\
\hline Chloramphenicol & 0.049 & 0.072 & 0.395 \\
\hline Clenbuterol hydrochloride & 0.049 & 0.091 & 0.097 \\
\hline Colchicine & 0.049 & 0.049 & 0.221 \\
\hline Cyhalothrin & 0.390 & 32.585 & 32.678 \\
\hline Cypermethrin & 0.390 & 101.691 & 102.766 \\
\hline Danofloxacin & 0.390 & 30.963 & 31.130 \\
\hline Dapsone & 0.049 & 0.071 & 0.371 \\
\hline Deltamethrin & 0.195 & 31.889 & 32.245 \\
\hline Dexamethasone & 0.049 & 0.383 & 0.417 \\
\hline Doramectin & 0.390 & 16.318 & 16.348 \\
\hline Eprinomectin & 0.390 & 20.963 & 21.364 \\
\hline Imidocarb & 0.390 & 50.858 & 51.062 \\
\hline Ivermectin & 0.390 & 10.665 & 10.949 \\
\hline Meloxicam & 0.195 & 16.055 & 16.382 \\
\hline Metronidazole & 0.049 & 0.072 & 0.087 \\
\hline Monensin sodium & 0.390 & 2.180 & 2.322 \\
\hline Procaine benzyl penicillin & 0.097 & 4.145 & 4.252 \\
\hline Sulfadimidine & 0.195 & 25.963 & 26.472 \\
\hline Trichlorfon & 0.390 & 51.141 & 51.434 \\
\hline Mycotoxins $^{1}(\mathrm{n}=22)$ & & & 0.000 \\
\hline DON & 0.390 & 0.439 & 0.501 \\
\hline FUS-X & 0.390 & 0.447 & 0.467 \\
\hline NEO & 0.390 & 0.397 & 0.419 \\
\hline $\mathrm{AFG}_{2}$ & 0.195 & 0.209 & 0.227 \\
\hline $\mathrm{AFG}_{1}$ & 0.195 & 0.202 & 0.215 \\
\hline $\mathrm{AFM}_{1}$ & 0.049 & 0.058 & 0.266 \\
\hline $\mathrm{AFB}_{2}$ & 0.097 & 0.105 & 0.111 \\
\hline $\mathrm{AFB}_{1}$ & 0.097 & 0.099 & 0.113 \\
\hline $\mathrm{HT} 2$ & 0.390 & 0.424 & 0.450 \\
\hline$\alpha-Z A L$ & 0.390 & 0.438 & 0.447 \\
\hline$\alpha-Z E L$ & 0.390 & 0.455 & 0.478 \\
\hline $\mathrm{AOH}$ & 0.390 & 0.428 & 0.451 \\
\hline $\mathrm{T} 2$ & 0.390 & 0.466 & 0.478 \\
\hline$\beta-Z A L$ & 0.390 & 0.435 & 0.457 \\
\hline$\beta-Z E L$ & 0.390 & 0.390 & 0.390 \\
\hline ZEN & 0.049 & 0.073 & 0.080 \\
\hline AME & 0.049 & 0.049 & 0.064 \\
\hline ENNB & 0.390 & 0.480 & 0.496 \\
\hline ENNB1 & 0.390 & 0.445 & 0.445 \\
\hline BEA & 0.390 & 0.488 & 0.520 \\
\hline ENNA1 & 0.390 & 0.458 & 0.524 \\
\hline ENNA & 0.390 & 0.431 & 0.500 \\
\hline
\end{tabular}

${ }^{1}$ Mycotoxin standards were as follows: aflatoxins $\left(\mathrm{AFM}_{1}, \mathrm{AFB}_{1}, \mathrm{AFB}_{2}, \mathrm{AFG}_{1}\right.$, and $\left.\mathrm{AFG}_{2}\right)$, deoxynivalenol (DON), HT2 toxin, T2 toxin, neosolaniol (NEO), fusarenon-X (FUS-X), zearalanol (ZEN), $\alpha$-zearalenol $(\alpha$-ZEL), $\beta$-zearalenol ( $\beta$-ZEL), $\alpha$-zearalanol ( $\alpha$-ZAL), $\beta$-zearalanol $(\beta$-ZAL), beauvericin (BEA), enniatins (ENNA, ENNA1, ENNB, and ENNB1), alternariol (AOH), and alternariolmonomethyl ether (AME).

samples, ranging from 0.3 to $88.6 \mathrm{ng} / \mathrm{mL}$. As regards ZEN, considered as not carcinogenic to humans and classified in the third group by IARC, no maximum limits are available in milk.

We detected ENN in up to 4 analyzed milk samples: ENNB $(22.2 \%, \mathrm{n}=12)$ at concentration ranges of 0.552 to $0.626 \mathrm{ng} / \mathrm{mL}$, and ENNA1 $(5.5 \%, \mathrm{n}=3)$ at concentrations of $0.903 \mathrm{ng} / \mathrm{mL}$. The ENN are emerging mycotoxins derived from Fusarium species, commonly found in cereal grains, animal feeds, and food commodities worldwide (Rodríguez-Carrasco et al., 2018; Pickova et al., 2020). Presently, ENNB is the most studied, because it has been the most often found in grains from European countries. The European Food Safety Authority has established that acute exposure to ENN does not indicate concern for human health. It 
Table 4. Occurrence of target compounds in 3 lots of 18 different brands of commercial infant milk formulas from southern Italy $(\mathrm{n}=54)$

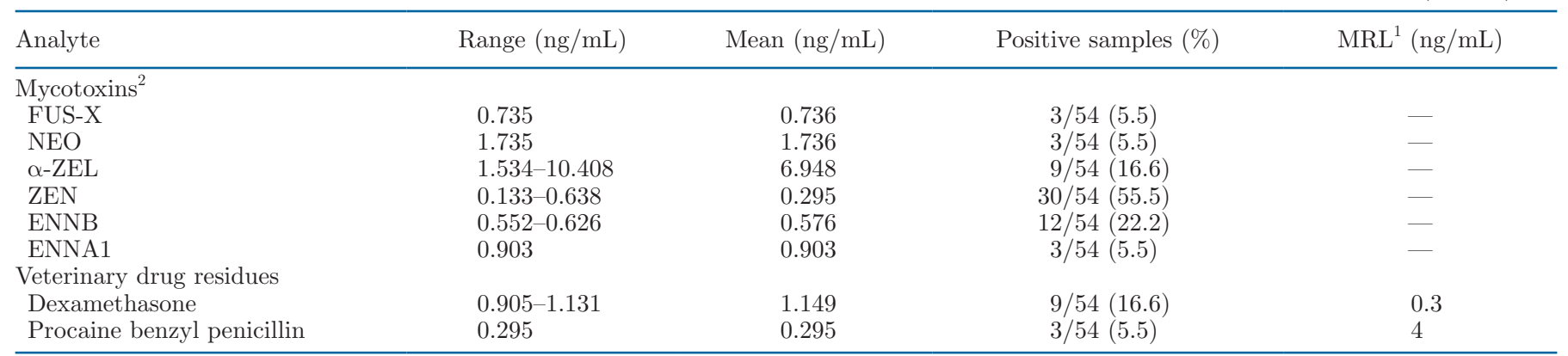

${ }^{1} \mathrm{MRL}=$ maximum residue level.

${ }^{2}$ Mycotoxin standards were as follows: neosolaniol (NEO), fusarenon-X (FUS-X), zearalenone (ZEN), $\alpha$-zearalenol ( $\alpha$-ZEL), and enniatins (ENNA1 and ENNB).

has not been possible to evaluate chronic exposure, due to overall lack of toxicity data (Rodríguez-Carrasco et al., 2020).

Retrospective Screening Analysis of Real Samples. The developed Q-Orbitrap HRMS strategy combines quantitative target analysis with identification of untargeted compounds. A retrospective approach was taken, without the need to re-run samples. Retrospective analysis of data enabled detection of untargeted contaminants in analyzed milk samples, although it was not possible to carry out a quantitative analysis based on a reference standard. The findings of retrospective data analysis are based only on a match of one precursor ion and do not include any product ion data. Results of untargeted mycotoxins $(\mathrm{n}=14)$ and veterinary drug residues $(\mathrm{n}=40)$ are reported in Figure 1 , whereas data on pesticides $(\mathrm{n}=283)$ are shown in Figure 2.

Data tentatively identified the occurrence of up to 5 mycotoxins and fungal metabolites, 11 pharmacologically active substances, and up to 49 different pesticides in the analyzed IMF. Regarding veterinary drugs, oxfendazole, mebendazone, and oxytetracycline were putatively identified in $5.5 \%$ samples. Similarly, oxfendazole was found in $2.7 \%$ Greek milk samples at an average concentration of $1 \mu \mathrm{g} / \mathrm{kg}$ (Dasenaki and Thomaidis, 2015). Aguilera-Luiz et al. (2012), reported oxfendazole, tilmicosin, trimethoprim, thiabendazole, and albendazole in $55.5 \%$ of commercial Spanish IMF $(\mathrm{n}=9)$. In our study, betamethasone and methylprednisolone were detected in $22.2 \%$ and $33.3 \%$ of samples, respectively. Corticosteroids are widely used veterinary drugs, often in combination with antimicrobial drugs. Although licensed for treatment of diseases in breeding animals, MRL for betamethasone and dexamethasone, prednisolone, and methylprednisolone have been set in the European Union at very low levels (EC, 2009).

Of special concern was the putative identification of decoquinate in $33.3 \%$ samples, although it is recom- mended not to use it in animals from which milk is produced for human consumption. Nebot et al. (2012) reported the occurrence of decoquinate in $0.8 \%$ analyzed Spanish milk samples, at a concentration of $5 \mu \mathrm{g} /$ $\mathrm{kg}$.

Ethoxyquin, fludioxonil, mepanipyrim, ethoxyquin, cyazofamid, acetamiprid, and triazophos were detected in $33.3 \%$ of the samples $(n=6)$. Aguilera-Luiz et al. (2011) reported detecting pesticides, including thiabendazole, acetamiprid, thiacloprid, carbendazim, thiophanate methyl, and bendiocarb, in milk. In particular, imidacloprid and atrazine desisopropyl were found in 4 milk samples at concentration levels higher than $10 \mu \mathrm{g} /$ $\mathrm{kg}$ in Aguilera-Luiz et al. (2011), although until now no MRL have been set for them in milk. Pesticides listed in Table 1 of Annex VIII of commission directive 2006/141/EC (EC, 2006a), not recommended in agricultural products intended for the production of IMF, were not tentatively found in analyzed samples.

Co-occurrence of pharmacologically active residues was found in a significant number of infant milk samples $(83.3 \%)$. Up to 4 pharmacologically active substances, decoquinate, dimetridazole, betamethasone, and oxfendazole, were detected simultaneously in one analyzed sample. Additive or synergistic effects of more contaminants coexisting in the same sample should be considered in risk assessment studies (Streit et al., 2013; Alassane-Kpembi et al., 2017; Klátyik et al., 2017). Given the large consumption of milk and milk derivates, a thorough investigation of the occurrence of contaminants, as well the adoption of measures to reduce their contamination of milk, is essential.

\section{Risk Characterization}

Risk characterization is an indispensable aspect to safeguard public health, which helps to identify risks threatening consumers. Therefore, it is extremely important to determine the percentage of tolerable daily 


\section{Retrospective analysis}

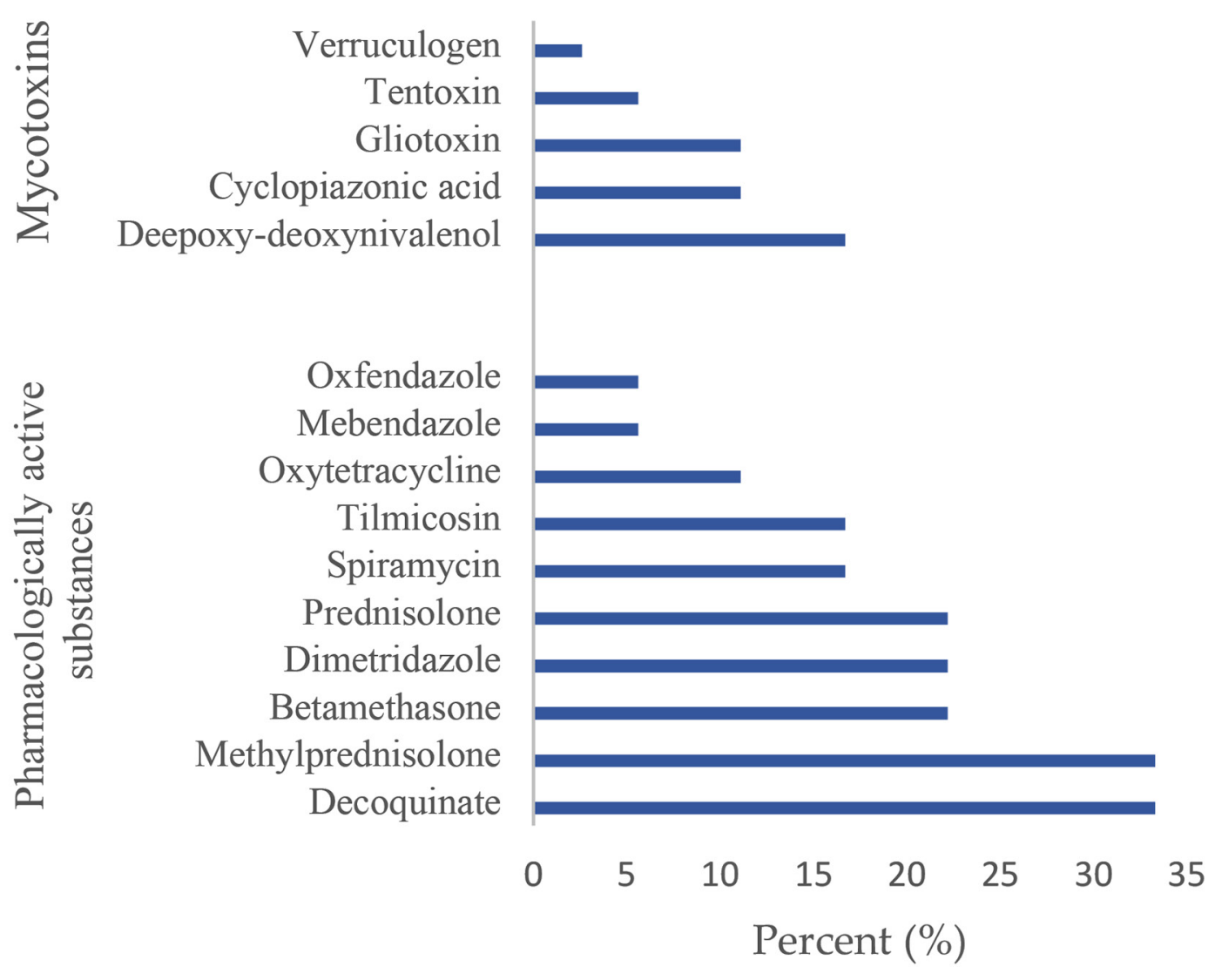

Figure 1. Percentages of samples and types of mycotoxins and pharmacologically active substances tentatively identified in commercial infant milk formulas, using retrospective analysis of data by matching the exact mass of theoretical precursor ions within $1 \mathrm{ppm}$.

intake (TDI) to avoid adverse effects due to dietary exposure. For calculation of TDI percentage, consumption data, body weight, and maximum concentration of each contaminant occurring in IMF were taken into consideration. As reported by the latest global individual food consumption data (FAO and WHO, 2021), Italian infant formula milk-based consumption is 22 and $6.91 \mathrm{~g} / \mathrm{d}$ for infants 1 to $3 \mathrm{yr}$ old and neonates 0 to 12 mo old, respectively, and body weight of 11.9 and $6.7 \mathrm{~kg}$ for infants 1 to $3 \mathrm{yr}$ old and neonates 0 to $12 \mathrm{mo}$ old, respectively, were evaluated (EFSA, 2012). Hence, to calculate TDI percentage, the following equation was used:

$$
\% \mathrm{TDI}=\frac{\mathrm{DI} \times \mathrm{C}}{\mathrm{TDI} \times \mathrm{BW} \times 1,000}
$$

where DI = daily substance intake $(\mathrm{ng} / \mathrm{mL}) ; \mathrm{C}=$ food consumption ( $\mathrm{kg}$ per capita per year); TDI $=$ tolerable daily intake $(\mu \mathrm{g} / \mathrm{kg}$ of $\mathrm{BW})$; and $\mathrm{BW}=$ body weight $(\mathrm{kg})$.

To achieve a more accurate estimation of exposure, 2 scenarios were established: the upper bound and the lower bound. For the upper bound, samples reported as negative or $<\mathrm{LOQ}$ were substituted by the LOQ, whereas the lower bound considered these samples as strictly negative. To estimate exposure, the mean concentration value was considered.

For risk characterization, TDI stated by the European Food Safety Authority was used. In the case of ZEN and its derived forms, TDI have been established for the sum of ZEN and its derived forms through a relative potency factors system according to their reported toxicity. The relative potency factors of $1,60,0.2$, and 1.5 are respectively assigned to ZEN, $\alpha$-ZEL, $\beta$-ZEL, and ZAN. Similarly, NEO is included in the TDI value established for $\mathrm{T} 2$ in the same relative potency factors system: T2, HT2, and NEO are given values of 1, 1, and 


\section{Retrospective analysis}

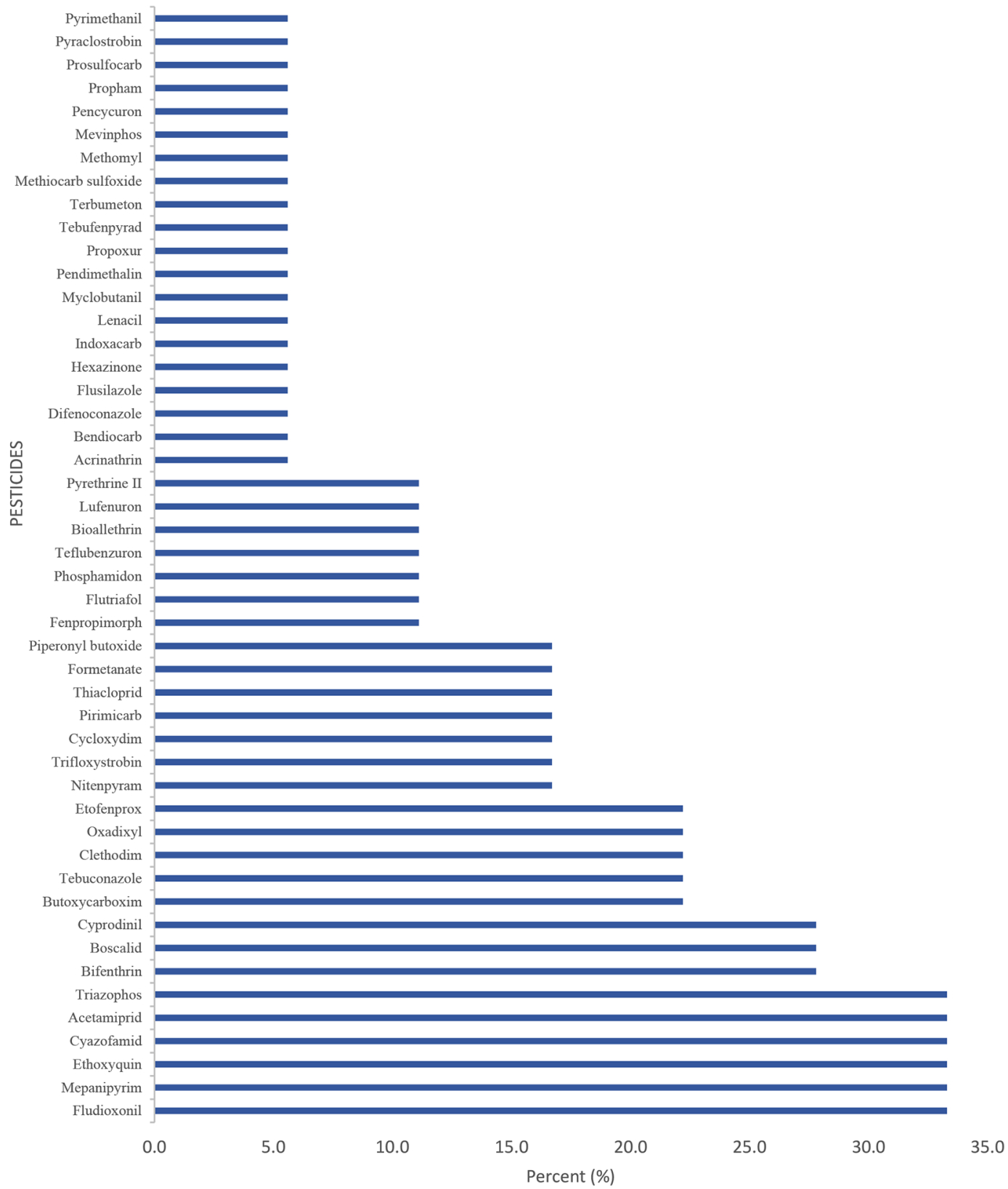

Figure 2. Percentages of samples and types of pesticides tentatively identified in commercial infant milk formulas, using retrospective analysis of data by matching the exact mass of theoretical precursor ions within $1 \mathrm{ppm}$.

0.3, respectively (EFSA, 2016, 2017). Results are shown in Table 5. Taking into consideration the lower bound, for a child 1 to $3 \mathrm{yr}$ old, the calculated TDI percentages are in a range of $0.28 \%$ to $16.18 \%$, whereas for infants 0 to 12 mo old, the calculated TDI percentages are in a range of $1.58 \%$ to $91.88 \%$. In the worst-case scenario represented by the upper bound, for a child 1 to $3 \mathrm{yr}$ old, the calculated TDI percentages are in a range of
$1.35 \%$ to $50.49 \%$, whereas for infants 0 to 12 mo old, the calculated TDI percentages are in a range of $7.70 \%$ to $286.77 \%$ Therefore, the European Food Safety Authority Scientific Committee (EFSA, 2012) does not recommend grouping infants from 0 to $3 \mathrm{yr}$, because their intake and body weights are variable, and instead suggested the evaluation of risk on a case-by-case basis. In the worst-case scenario, the percentage found does 
Table 5. Exposure assessment for mycotoxins and pharmacologically active substances exposure in infants calculated on the consumption of infant food according to the latest FAO data in Italy (FAO, 2018)

\begin{tabular}{|c|c|c|c|c|c|c|c|}
\hline \multirow[b]{2}{*}{ Analyte } & \multicolumn{2}{|c|}{$\begin{array}{c}\text { Concentration }^{1} \\
(\mu \mathrm{g} / \mathrm{kg})\end{array}$} & \multirow[b]{2}{*}{$\begin{array}{c}\mathrm{TDI}^{2} \\
(\mu \mathrm{g} / \mathrm{kg} \text { of } \mathrm{BW})\end{array}$} & \multicolumn{2}{|c|}{$\begin{array}{l}\% \text { TDI, infants } \\
\text { aged } 0-12 \mathrm{mo}^{3}\end{array}$} & \multicolumn{2}{|c|}{$\begin{array}{c}\% \text { TDI, infants } \\
\text { aged } 1-3 \mathrm{yr}^{3}\end{array}$} \\
\hline & $\begin{array}{l}\text { Upper } \\
\text { bound }\end{array}$ & $\begin{array}{l}\text { Lower } \\
\text { bound }\end{array}$ & & $\begin{array}{l}\text { Upper } \\
\text { bound }\end{array}$ & $\begin{array}{l}\text { Lower } \\
\text { bound }\end{array}$ & $\begin{array}{l}\text { Upper } \\
\text { bound }\end{array}$ & $\begin{array}{l}\text { Lower } \\
\text { bound }\end{array}$ \\
\hline \multicolumn{8}{|l|}{ Mycotoxins $^{4}$} \\
\hline FUS-X & 0.409 & 0.041 & None established & - & - & & - \\
\hline $\mathrm{NEO}+\mathrm{T} 2+\mathrm{HT} 2$ & 0.465 & 0.096 & 0.02 & 76.69 & 1.58 & 1.35 & 0.28 \\
\hline ENNA1 & 0.422 & 0.050 & None established & - & - & - & - \\
\hline \multicolumn{8}{|l|}{ Veterinary drug residues } \\
\hline Dexamethasone & 0.350 & 0.192 & $0-0.015^{5}$ & 7.70 & 4.22 & 1.36 & 0.74 \\
\hline Procaine benzyl penicillin & 0.108 & 0.016 & None established & & - & - & - \\
\hline
\end{tabular}

${ }^{1}$ Highest residue concentration detected in the tested samples.

${ }^{2} \mathrm{TDI}=$ tolerable daily intake, expressed as $\mu \mathrm{g} / \mathrm{kg}$ of BW.

${ }^{3} 1$ - to 3 -yr-old child with $11.9 \mathrm{~kg}$ of BW; 0- to 12-mo-old child with $6.7 \mathrm{~kg}$ of BW (EFSA, 2012). Dashes indicate that no TDI was established for a given analyte.

${ }^{4}$ Mycotoxin standards were as follows: fusarenon-X (FUS-X), neosolaniol (NEO), zearalenone (ZEN), $\alpha$-zearalenol ( $\alpha$-ZEL), and enniatins (ENNA1 and ENNB).

${ }^{5}$ Percent TDI is shown for a TDI of 0.015 .

not represent a health hazard but a significant contribution to TDI. For some contaminants, TDI is not established, making it impossible to assess the risk of exposure.

As expected, infants are the most vulnerable category of the population, considering the high infant milk formula intake compared with older children and adolescents in relation to their low body weight. Based on risk assessment, a smaller quantity of contaminant residues could be enough to provoke adverse effects on infants. Hence, it is highly recommended to have a watchful attitude regarding food intended for susceptible groups. The total process of infant milk formula manufacturing should be controlled to ensure the use of raw materials with acceptable MRL.

\section{CONCLUSIONS}

This work proposed a multi-class analysis of mycotoxins $(\mathrm{n}=22)$ and veterinary drug residues $(\mathrm{n}=23)$, in addition to retrospective analysis of other contaminants for which analytical standards $(\mathrm{n}=54)$ are not available and pesticides $(\mathrm{n}=283)$ in IMF. Advantages of the validated method include a single extraction for all the studied analytes, rapid determination, simple sample pretreatment, and high sensitivity. Results of evaluated parameters are in accordance with the limits reported by European Commission regulations. The validated method was applied to the analysis of 3 lots of 18 different brands of IMF. Results showed occurrence of contaminant residues in analyzed IMF: dexamethasone, procaine benzyl penicillin, zearalenone, and $\alpha$-zearalenol. Up to 65 contaminants were tentatively identified via retrospective analysis based on the mass spectral library. Orbitrap technology represents a practical tool for sure and precise identification of a wide number of contaminants in a 9-min analytical runtime. Moreover, the in-house validated method could be proposed for simultaneous identification of contaminants in routine analysis of IMF, reassuring consumers on the safety of purchased foods.

\section{ACKNOWLEDGMENTS}

This research was supported by the Spanish Ministry of Science and Innovation Project (PID2020-115871RBI00). The authors have not stated any conflicts of interest.

\section{REFERENCES}

Aguilera-Luiz, M. M., P. Plaza-Bolaños, R. Romero-González, J. L. Martínez Vidal, and A. Garrido Frenich. 2011. Comparison of the efficiency of different extraction methods for the simultaneous determination of mycotoxins and pesticides in milk samples by ultra high-performance liquid chromatography-tandem mass spectrometry. Anal. Bioanal. Chem. 399:2863-2875. https://doi.org/10 .1007/s00216-011-4670-7.

Aguilera-Luiz, M. M., J. L. Martínez Vidal, R. Romero-González, and A. Garrido Frenich. 2012. Multiclass method for fast determination of veterinary drug residues in baby food by ultra-high-performance liquid chromatography-tandem mass spectrometry. Food Chem. 132:2171-2180. https://doi.org/10.1016/j.foodchem.2011.12.042.

Akhtar, S., and K. Ahad. 2017. Pesticides residue in milk and milk products: Mini review. Pak. J. Anal. Environ. Chem. 18:37-45. https://doi.org/10.21743/pjaec/2017.06.03.

Alassane-Kpembi, I., G. Schatzmayr, I. Taranu, D. Marin, O. Puel, and I. P. Oswald. 2017. Mycotoxins co-contamination: Method- 
ological aspects and biological relevance of combined toxicity studies. Crit. Rev. Food Sci. Nutr. 57:3489-3507. https://doi.org/10 $.1080 / 10408398.2016 .1140632$.

Awaisheh, S. S., R. J. Rahahleh, R. M. Algroom, A. A. Al-Bakheit, J. M. Al-Khaza'leh, and B. A. Al-Dababseh. 2019. Contamination level and exposure assessment to Aflatoxin M1 in Jordanian infant milk formulas. Ital. J. Food Saf. 8. https://doi.org/10.4081/ijfs .2019 .8263

Bacanll, M., and N. Başaran. 2019. Importance of antibiotic residues in animal food. Food Chem. Toxicol. 125:462-466. https://doi.org/ 10.1016/j.fct.2019.01.033.

Barreiro, J. C., A. L. Luiz, S. C. F. Maciel, E. V. S. Maciel, and F. M. Lanças. 2015. Recent approaches for on-line analysis of residues and contaminants in food matrices: A review. J. Sep. Sci. 38:1721-1732. https://doi.org/10.1002/jssc.201401285.

Becker-Algeri, T. A., D. Castagnaro, K. de Bortoli, C. de Souza, D. A. Drunkler, and E. Badiale-Furlong. 2016. Mycotoxins in bovine milk and dairy products: A review. J. Food Sci. 81:R544-R552. https://doi.org/10.1111/1750-3841.13204.

Benkerroum, N. 2016. Mycotoxins in dairy products: A review. Int. Dairy J. 62:63-75. https://doi.org/10.1016/j.idairyj.2016.07.002.

Brodehl, A., A. Möller, H.-J. Kunte, M. Koch, and R. Maul. 2014. Biotransformation of the mycotoxin zearalenone by fungi of the genera Rhizopus and Aspergillus. FEMS Microbiol. Lett. 359:124-130. https://doi.org/10.1111/1574-6968.12586.

Castaldo, L., L. Izzo, S. De Pascale, A. Narváez, Y. Rodriguez-Carrasco, and A. Ritieni. 2021. Chemical composition, in vitro bioaccessibility and antioxidant activity of polyphenolic compounds from nutraceutical fennel waste extract. Molecules 26:1968. https://doi .org/10.3390/molecules26071968.

Castaldo, L., A. Narváez, L. Izzo, G. Graziani, and A. Ritieni. 2020. In vitro bioaccessibility and antioxidant activity of coffee silverskin polyphenolic extract and characterization of bioactive compounds using UHPLC-Q-Orbitrap HRMS. Molecules 25:2132. https://doi .org $/ 10.3390 /$ molecules25092132.

Chung, S. W., and C.-H. Lam. 2015. Development of a 15-class multiresidue method for analyzing 78 hydrophilic and hydrophobic veterinary drugs in milk, egg and meat by liquid chromatographytandem mass spectrometry. Anal. Methods 7:6764-6776. https:// doi.org/10.1039/C5AY01317H.

Codex Alimentarius. 2016. International foods standards: Standard for infant formula and formulas for special medical purposes intended for infants, CODEX STAN 72-1981. Amendment: 1983, 1985, 1987, 2011, 2015 and 2016. Revision: 2007. http://www.fao .org/fao-who-codexalimentarius/sh-proxy/en/?lnk=1\&url=https $\% 253 \mathrm{~A} \% 252 \mathrm{~F} \% 252 \mathrm{~F}$ workspace.fao.org $\% 252 \mathrm{Fsites} \% 252 \mathrm{Fcodex}$ \%252FStandards\%252FCXS\%2B72-1981\%252FCXS_072e.pdf.

Danezis, G. P., C. J. Anagnostopoulos, K. Liapis, and M. A. Koupparis. 2016. Multi-residue analysis of pesticides, plant hormones, veterinary drugs and mycotoxins using HILIC chromatographyMS/MS in various food matrices. Anal. Chim. Acta 942:121-138. https://doi.org/10.1016/j.aca.2016.09.011.

Dasenaki, M. E., and N. S. Thomaidis. 2015. Multi-residue determination of 115 veterinary drugs and pharmaceutical residues in milk powder, butter, fish tissue and eggs using liquid chromatographytandem mass spectrometry. Anal. Chim. Acta 880:103-121. https: //doi.org/10.1016/j.aca.2015.04.013.

de Mendonça Pereira, B. F., C. C. de Almeida, K. C. Leandro, M. P. da Costa, C. A. Conte-Junior, and B. F. Spisso. 2020. Occurrence, sources, and pathways of chemical contaminants in infant formulas. Compr. Rev. Food Sci. Food Saf. 19:1378-1396. https://doi .org/10.1111/1541-4337.12559.

EC (European Commission). 2002. Commission Decision of $12 \mathrm{Au}-$ gust 2002 implementing Council Directive 96/23/EC concerning the performance of analytical methods and the interpretation of results. https://eur-lex.europa.eu/legal-content/EN/ALL/?uri= CELEX\%3A32002D0657.

EC (European Commission). 2004. Commission Directive 2004/28/ EC of the European Parliament and the council of 31 March 2004 amending Directive 2001/82/EC on the Community code relating to veterinary medicinal products. https://ec.europa.eu/health/ sites/default/files/files/eudralex/vol-5/dir_2004_28/dir_2004_28 _en.pdf.

EC (European Commission). 2006a. Commission Directive 2006/141/ EC of 22 December 2006 on infant formulae and follow-on formulae and amending Directive 1999/21/EC. https://eur-lex.europa .eu/legal-content/en/ALL/?uri=CELEX\%3A32006L0141.

EC (European Commission). 2006b. Commission Regulation (EC) No. 1881/2006 setting maximum levels for certain contaminants in foodstuffs. https://eur-lex.europa.eu/legal-content/EN/ALL/?uri $=$ CELEX\%3A32006R1881.

EC (European Commission). 2006c. Commission Regulation 401/2006 of 23 February 2006 laying down the methods of sampling and analysis for the official control of the levels of mycotoxins in foodstuffs. https://eur-lex.europa.eu/legal-content/IT/ALL/?uri= CELEX\%3A32006R0401.

EC (European Commission). 2008. Commission Regulation (EC) No. 839/2008 of 31 July 2008 amending Regulation (EC) No 396/2005 of the European Parliament and of the Council as regards Annexes II, III and IV on maximum residue levels of pesticides in or on certain products (Text with EEA relevance). https://eur-lex.europa .eu/legal-content/EN/TXT/?uri=CELEX:32008R0839.

EC (European Commission). 2009. Commission Regulation (EU) No. $37 / 2010$ of 22 December 2009 on pharmacologically active substances and their classification regarding maximum residue limits in foodstuffs of animal origin. https://eur-lex.europa.eu/legal -content/EN/TXT/?uri=celex\%3A32010R0037.

EFSA (European Food Safety Authority). 2012. Guidance on selected default values to be used by the EFSA Scientific Committee, Scientific Panels and Units in the absence of actual measured data. EFSA J. 10:2579.

EFSA (European Food Safety Authority). 2016. Appropriateness to set a group health-based guidance value for zearalenone and its modified forms. EFSA J. 14:4425.

EFSA (European Food Safety Authority). 2017. Appropriateness to set a group health based guidance value for T2 and HT2 toxin and its modified forms. EFSA J. 15:4655.

European Commission Health and Food Safety Directorate General. 2016. Guidance document on identification of mycotoxin in food and feed. SANTE/12089/2016. European Commission SANTE.

FAO (Food and Agriculture Organization). 2018. Food balance sheet. Accessed Oct. 20, 2021. https://www.fao.org/faostat/en/\#data/ FBS.

FAO-WHO (Food and Agriculture Organization-World Health Organization). 2021. Global Individual Food consumption data Tool (GIFT): Italy, INRAN SCAI 2005-2006, CREA, Alimenti e Nutrizione. Accessed Oct. 20, 2021. https://www.fao.org/gift -individual-food-consumption/data-and-indicator/en/.

Gómez-Pérez, M. L., R. Romero-González, V. José Luis Martínez, and A. Garrido Frenich. 2015. Analysis of pesticide and veterinary drug residues in baby food by liquid chromatography coupled to Orbitrap high resolution mass spectrometry. Talanta 131:1-7. https://doi.org/10.1016/j.talanta.2014.07.066.

González-Curbelo, M., B. Socas-Rodríguez, A. Herrera-Herrera, J. González-Sálamo, J. Hernández-Borges, and M. Rodríguez-Delgado. 2015. Evolution and applications of the QuEChERS method. Trends Analyt. Chem. 71:169-185. https://doi.org/10.1016/j.trac .2015.04.012.

Guan, S., M. Gong, Y. Yin, R. Huang, Z. Ruan, T. Zhou, and M. Xie. 2011. Occurrence of mycotoxins in feeds and feed ingredients in China. J. Food Agric. Environ. 9:163-167.

Haque, M. A., Y. Wang, Z. Shen, X. Li, M. K. Saleemi, and C. J. M. He. 2020. Mycotoxin contamination and control strategy in human, domestic animal and poultry: A review. Microb. Pathog. 142:104095. https://doi.org/10.1016/j.micpath.2020.104095.

IARC (International Agency for Research on Cancer). 2012. Agents Classified by the IARC Monographs. Vol. 1. IARC.

Izzo, L., Y. Rodríguez-Carrasco, J. Tolosa, G. Graziani, A. Gaspari, and A. Ritieni. 2020. Target analysis and retrospective screening of mycotoxins and pharmacologically active substances in milk using an ultra-high-performance liquid chromatography/high-resolution 
mass spectrometry approach. J. Dairy Sci. 103:1250-1260. https:/ /doi.org/10.3168/jds.2019-17277.

Jia, W., X. Chu, Y. Ling, J. Huang, and J. Chang. 2014. Highthroughput screening of pesticide and veterinary drug residues in baby food by liquid chromatography coupled to quadrupole Orbitrap mass spectrometry. J. Chromatogr. A 1347:122-128. https:// doi.org/10.1016/j.chroma.2014.04.081.

Kaufmann, A. 2014. Combining UHPLC and high-resolution MS: A viable approach for the analysis of complex samples? Trends Analyt. Chem. 63:113-128. https://doi.org/10.1016/j.trac.2014.06.025.

Klátyik, S., P. Bohus, B. Darvas, and A. Székács. 2017. Authorization and toxicity of veterinary drugs and plant protection products: Residues of the active ingredients in food and feed and toxicity problems related to adjuvants. Front. Vet. Sci. 4:146. https://doi .org/10.3389/fvets.2017.00146.

Lehotay, S. J., A. Kok, M. Hiemstra, and P. Bodegraven. 2005. Validation of a fast and easy method for the determination of residues from 229 pesticides in fruits and vegetables using gas and liquid chromatography and mass spectrometric detection. J. AOAC Int. 88:595-614. https://doi.org/10.1093/jaoac/88.2.595.

Li, R., X. Wang, T. Zhou, D. Yang, Q. Wang, and Y. Zhou. 2014. Occurrence of four mycotoxins in cereal and oil products in Yangtze Delta region of China and their food safety risks. Food Control 35:117-122. https://doi.org/10.1016/j.foodcont.2013.06.042.

López-Ruiz, R., R. Romero-González, and A. G. Frenich. 2019. Ultrahigh-pressure liquid chromatography-mass spectrometry: An overview of the last decade. Trends Analyt. Chem. 118:170-181.

Masum, A., J. Chandrapala, T. Huppertz, B. Adhikari, and B. Zisu. 2021. Production and characterization of infant milk formula powders: A review. Dry. Technol. 39:1492-1512. https://doi.org/10 $.1080 / 07373937.2020 .1767645$.

Mol, H. G., P. Plaza-Bolaños, P. Zomer, T. C. de Rijk, A. A. Stolker, and P. P. Mulder. 2008. Toward a generic extraction method for simultaneous determination of pesticides, mycotoxins, plant toxins, and veterinary drugs in feed and food matrixes. Anal. Chem. 80:9450-9459. https://doi.org/10.1021/ac801557f.

Narváez, A., Y. Rodríguez-Carrasco, L. Castaldo, L. Izzo, G. Graziani, and A. J. M. Ritieni. 2020a. Occurrence and exposure assessment of mycotoxins in ready-to-eat tree nut products through ultra-high performance liquid chromatography coupled with high resolution Q-Orbitrap mass spectrometry. Metabolites 10:344. https://doi .org/10.3390/metabo10090344.

Narváez, A., Y. Rodríguez-Carrasco, L. Izzo, L. Castaldo, and A. Ritieni. 2020b. Target quantification and semi-target screening of undesirable substances in pear juices using ultra-high-performance liquid chromatography-quadrupole orbitrap mass spectrometry. Foods 9:841. https://doi.org/10.3390/foods9070841.

Nebot, C., A. Iglesias, P. Regal, J. Miranda, A. Cepeda, and C. Fente. 2012. Development of a multi-class method for the identification and quantification of residues of antibiotics, coccidiostats and corticosteroids in milk by liquid chromatography-tandem mass spectrometry. Int. Dairy J. 22:78-85. https://doi.org/10.1016/j.idairyj .2011.09.001.

Neme, K., and A. Mohammed. 2017. Mycotoxin occurrence in grains and the role of postharvest management as a mitigation strategies. A review. Food Control 78:412-425. https://doi.org/10.1016/ j.foodcont.2017.03.012.

Ogunade, I. M., C. Martinez-Tuppia, O. C. M. Queiroz, Y. Jiang, P. Drouin, F. Wu, D. Vyas, and A. T. Adesogan. 2018. Silage review: Mycotoxins in silage: Occurrence, effects, prevention, and mitigation. J. Dairy Sci. 101:4034-4059. https://doi.org/10.3168/ jds.2017-13788.

Pickova, D., V. Ostry, J. Toman, and F. Malir. 2020. Presence of mycotoxins in milk thistle (Silybum marianum) food supple- ments: A review. Toxins (Basel) 12:782. https://doi.org/10.3390/ toxins 12120782

Pleadin, J., A. Vulić, M. Zadravec, T. Lešić, M. Benić, V. Jaki Tkalec, and N. Vahčić. 2017. Presence of Fusarium mycotoxins in feedstuffs and cow milk sampled from Croatian farms during 2015. Mljekarstvo: časopis za unaprjeđenje proizvodnje i prerade mlijeka 67:102-111. https://doi.org/10.15567/mljekarstvo.2017.0202.

Rejczak, T., and T. Tuzimski. 2015. A review of recent developments and trends in the QuEChERS sample preparation approach. Open Chem. 13. https://doi.org/10.1515/chem-2015-0109.

Rodríguez-Carrasco, Y., L. Izzo, A. Gaspari, G. Graziani, J. Mañes, and A. Ritieni. 2018. Urinary levels of enniatin B and its phase I metabolites: First human pilot biomonitoring study. Food Chem. Toxicol. 118:454-459. https://doi.org/10.1016/j.fct.2018.05.052.

Rodríguez-Carrasco, Y., A. Narváez, L. Izzo, A. Gaspari, G. Graziani, and A. Ritieni. 2020. Biomonitoring of enniatin B1 and its phase I metabolites in human urine: First large-scale study. Toxins (Basel) 12:415. https://doi.org/10.3390/toxins12060415.

Rossi, R., G. Saluti, S. Moretti, I. Diamanti, D. Giusepponi, and R. Galarini. 2018. Multiclass methods for the analysis of antibiotic residues in milk by liquid chromatography coupled to mass spectrometry: A review. Food Addit. Contam. Part A Chem. Anal Control Expo. Risk Assess. 35:241-257. https://doi.org/10.1080/ 19440049.2017.1393107.

Samsidar, A., S. Siddiquee, and S. M. Shaarani. 2018. A review of extraction, analytical and advanced methods for determination of pesticides in environment and foodstuffs. Trends Food Sci. Technol. 71:188-201. https://doi.org/10.1016/j.tifs.2017.11.011.

Shamay, A., F. Shapiro, H. Barash, I. Bruckental, and N. Silanikove. 2000. Effect of dexamethasone on milk yield and composition in dairy cows. Pages 343-352 in Proc. Annales de zootechnie. EDP Sciences.

Streit, E., C. Schwab, M. Sulyok, K. Naehrer, R. Krska, and G. Schatzmayr. 2013. Multi-mycotoxin screening reveals the occurrence of 139 different secondary metabolites in feed and feed ingredients. Toxins (Basel) 5:504-523. https://doi.org/10.3390/ toxins5030504.

Thompson, L. A., and W. S. Darwish. 2019. Environmental chemical contaminants in food: Review of a global problem. J. Toxicol. 2019:1-14. https://doi.org/10.1155/2019/2345283.

UNICEF. 2019. Breastfeeding. Accessed Oct. 20, 2021. https://www .unicef.org/nutrition/index_24763.html.

Ushkalov, V., V. Danchuk, S. Midyk, N. Voloshchuk, and O. Danchuk. 2020. Mycotoxins in milk and in dairy products. J. Food Sci. Technol. 14:137-149. https://doi.org/10.15673/fst.v14i3.1786.

Wittenberg, J. B., K. A. Simon, and J. W. Wong. 2017. Targeted multiresidue analysis of veterinary drugs in milk-based powders using liquid chromatography-tandem mass spectrometry (LC-MS/MS). J. Agric. Food Chem. 65:7288-7293. https://doi.org/10.1021/acs .jafc.6b05263.

Zhan, J., X. Yu, Y.-y. Zhong, Z. Zhang, X. Cui, J. Peng, R. Feng, X. Liu, and Y. Zhu. 2012. Generic and rapid determination of veterinary drug residues and other contaminants in raw milk by ultra performance liquid chromatography-tandem mass spectrometry. J. Chromatogr. B Analyt. Technol. Biomed. Life Sci. 906:48-57. https://doi.org/10.1016/j.jchromb.2012.08.018.

Zhou, J., J.-J. Xu, J.-M. Cong, Z.-X. Cai, J.-S. Zhang, J.-L. Wang, and Y.-P. Ren. 2018. Optimization for quick, easy, cheap, effective, rugged and safe extraction of mycotoxins and veterinary drugs by response surface methodology for application to egg and milk. J. Chromatogr. A 1532:20-29. https://doi.org/10.1016/j.chroma .2017.11.050. 